\title{
Periods in Extensions of Words
}

Abstract Let $\pi(w)$ denote the minimum period of the word $w$. Let $w$ be a primitive word with period $\pi(w)<|w|$, and $z$ a prefix of $w$. It is shown that if $\pi(w z)=$ $\pi(w)$, then $|z|<\pi(w)-\operatorname{gcd}(|w|,|z|)$. Detailed improvements of this result are also proven. As a corollary we give a short proof of the fact that if $u, v, w$ are primitive words such that $u^{2}$ is a prefix of $v^{2}$, and $v^{2}$ is a prefix of $w^{2}$, then $|w|>2|u|$. Finally, we show that each primitive word $w$ has a conjugate $w^{\prime}=v u$, where $w=u v$, such that $\pi\left(w^{\prime}\right)=\left|w^{\prime}\right|$ and $|u|<\pi(w)$.

\section{Introduction}

Various aspects of periodicity play a central rôle in combinatorics on words and its applications; see Lothaire's books [8-10]. The notion of periodicity is well posed in many problems concerning algorithmic aspects of strings: in pattern matching, compression of strings, sequence analysis, and so forth.

In this paper we study extensions of words with respect to their periodicity. Let $w$ be a word over a finite alphabet $A$. The length of $w$ is denoted by $|w|$. The empty word is denoted by $\varepsilon$. A positive integer $p$ is a period of $w$, if $w=(u v)^{k} u$ where $p=|u v|, k \geq 1$, and $v \neq \varepsilon$. The minimum period of $w$ is denoted by $\pi(w)$.

For a word $w=u v$, the word $u$ is a prefix of $w$, denoted by $u \leq_{\mathrm{p}} w$, and $v$ is a suffix of $w$, denoted by $v \leq_{\mathrm{s}} w$. If $v$ is nonempty, then $u$ is a proper prefix of $w$, denoted by $u<_{\mathrm{p}} w$. A nonempty word $u$ is a border of $w$, if $u$ is a prefix and a suffix of $w$, i.e., $u x=w=y u$ for some nonempty words $x$ and $y$. Each word has a unique factorization in the form $w=u^{k} v$, where $k \geq 1, v<_{\mathrm{p}} u$ and $|u|=\pi(w)$. Here $u$ is called the root of $w$ and $v$ the residue of $w$. We denote the length $|v| \geq 0$ of the residue $v$ by $\rho(w)$.

Department of Mathematics, University of Turku, 20014 Turku, Finland, E-mail: harju@utu.fi Institute of Formal Methods in Computer Science, University of Stuttgart, 70569 Stuttgart, Germany, E-mail: nowotka@ fmi.uni-stuttgart.de 
A word is primitive if it is not a power of a shorter word, i.e., if $\pi(w)$ does not divide $|w|$ properly.

Let $w$ be a word with a nonempty residue and a prefix $z \leq_{\mathrm{p}} w$. We show that if the word $w z$ has the same minimum period as $w$, that is, $\pi(w z)=\pi(w)$, then $|z|<\pi(w)-\operatorname{gcd}(|w|,|z|)$, where gcd denotes the greatest common divisor function. As a corollary we give a short proof of the well known result due to Crochemore and Rytter [4] stating that if $u, v, w$ are primitive words such that $u^{2}<_{\mathrm{p}}$ $v^{2}<_{\mathrm{p}} w^{2}$, then $u^{2}<_{\mathrm{p}} w$, i.e., $|w|>2|u|$. Finally, we strengthen the above extension result by showing that if $w$ is a word with $u$ as a root and $w$ has a nonempty residue, then $\pi(w z)>\pi(w)$ for all prefixes $z \leq_{\mathrm{p}} w$ with $|z| \geq \pi(w)+\pi(u)-\rho(w)-1$.

In the last section, we study extensions $w z$ that force the period $\pi(w z)=|w|$. This problem is stated for unbordered conjugates. For this, let $\tau(w)$ denote the shortest prefix of the word $w$, say $w=\tau(w) u$, such that the conjugate $u \tau(w)$ is unbordered, i.e., $\pi(u \tau(w))=|u \tau(w)|$. We show that for each primitive word $w$ it holds that $\tau(w)<\pi(w)$.

\section{Extensions of words by periods}

It is clear that if $u$ is a border of a word $w$, then $|w|-|u|$ is a period of $w$, and thus $|w|-|u| \geq \pi(w)$. A word $w$ is said to be bordered (or self-correlated [11]), if it has a border, that is, if $w$ has a prefix of length less than $|w|$ which is also a suffix of $w$. If $w$ is not bordered, it is called unbordered. Clearly, a word $w$ is unbordered if and only if $\pi(w)=|w|$.

We begin with an application of the basic periodicity result of Fine and Wilf [6]:

Theorem 1 (Fine and Wilf) If a word $w$ has two periods $p$ and $q$ such that $|w| \geq$ $p+q-\operatorname{gcd}(p, q)$, then also $\operatorname{gcd}(p, q)$ is a period of $w$.

Note that if $w$ has an empty residue, then $\pi(w z)=\pi(w)$ for all words $z=w^{k} u$ with $u \leq_{\mathrm{p}} w$ and $k \geq 0$. Therefore, in the sequel we consider words with nonempty residues. Note that each word $w$ with a nonempty residue is primitive, and thus $\pi\left(w^{2}\right)=|w|>\pi(w)$.

Theorem 2 Let $w$ be a word with a nonempty residue and a prefix $z \leq_{\mathrm{p}} w$.

$$
\text { If } \pi(w z)=\pi(w) \text { then }|z|<\pi(w)-\operatorname{gcd}(\pi(w),|w|) .
$$

Proof Clearly $\pi(w z) \geq \pi(w)$. Let $d=\operatorname{gcd}(\pi(w),|w|)$, and suppose that $z \leq_{\mathrm{p}} w$ satisfies $\pi(w z)=\pi(w)$. Then both $|w|$ and $\pi(w)$ are different periods of $w z$. If $|w z| \geq \pi(w)+|w|-d$, then Theorem 1 implies that $d$ is a period of $w z$. In this case, $d=\pi(w)$, since $\pi(w z) \geq \pi(w)$, and so $\pi(w)$ divides $|w|$ contradicting primitivity of $w$; hence the claim follows.

The following example shows that the bound given in Theorem 2 is optimal for all lengths.

Example 1 Consider the word

$$
w=a^{n-1} b a
$$

with the minimum period $\pi(w)=n$, and let $z=a^{n-2} \leq_{\mathrm{p}} w$. We have $\pi(w z)=n$, where $|z|=|w|-3=\pi(w)-\operatorname{gcd}(\pi(w),|w|)-2$, since $\operatorname{gcd}(n, n+1)=1$. 
The following example shows that the condition $|z| \geq \pi(w)-\operatorname{gcd}(\pi(w),|w|)$ does not imply that $\pi(w z)=|w|$.

Example 2 Consider the word

$$
w=a b a b a a b a b .
$$

Then $\pi(w)=|a b a b a|=5$. Let $z=a b a$. We have $|z|=\pi(w)-2$ and

$$
w z=a b a b a \cdot a b a b \cdot a b a
$$

with $\pi(w)=5<7=\pi(w z)<9=|w|$, since $|a b a b a a b|$ is a period of $w z$.

The following result is due to Crochemore and Rytter [4]. A short proof due to Diekert is given in [9, Lemma 8.1.14]. Below we show that this result follows from Theorem 2 . Note that an integer $p \leq|w|$ is a period of the word $w$ if and only if $w \leq_{\mathrm{p}} x w$, where $x \leq_{\mathrm{p}} w$ is such that $|x|=p$.

Corollary 1 Let $u, v, w$ be primitive words with $u^{2}<_{\mathrm{p}} v^{2}<_{\mathrm{p}} w^{2}$. Then $|w|>2|u|$.

Proof Suppose that $|w| \leq 2|u|$, and thus $w<_{\mathrm{p}} v^{2}<_{\mathrm{p}} w^{2}$. Hence $w$ has a nonempty residue. Let $w=v x$. Then $|x|$ is a period of $v$, since $v v \leq_{\mathrm{p}} w w=v x v x$ and so $v \leq_{\mathrm{p}} x v$. Now $\pi(v) \leq|x|$, and, by Theorem $2, \pi(w) \geq|v|$, and so $\pi(w)=|v|$. However, also $|u|$ is a period of $w$, since $w<_{\mathrm{p}} u^{2}$. Therefore $|v|=\pi(w)=|u|$ gives a contradiction.

For a word $w$ with a nonempty residue, let its maximal extension number be defined by

$$
\kappa(w)=\max \left\{p|p=| z \mid \text { for a prefix } z \leq_{\mathrm{p}} w \text { with } \pi(w z)=\pi(w)\right\} .
$$

Theorem 2, $\kappa(w)$ exists and satisfies $\kappa(w)<\pi(w)-1$. For a nonempty word $w$, let $w^{\bullet}$ denote the word from which the last letter is removed. For the proof of the following result, see Berstel and Karhumäki [1].

Lemma 1 Let $u$ and $v$ be two nonempty words. If $u v^{\bullet}=v u^{\bullet}$ then there exists a word $g$ such that $u=g^{i}$ and $v=g^{j}$ for some $i, j \geq 1$.

We shall now have a partial improvement of Theorem 2 .

Theorem 3 Let $w$ be a word with a nonempty residue and let $u$ be the root of $w$. Then

$$
\kappa(w) \leq \pi(w)+\pi(u)-\rho(w)-2 .
$$

Proof Let $u=v y$ where $|v|=\rho(w)$, and let $x$ be the root of $u$. Assume that there exists a prefix $z \leq_{\mathrm{p}} w$ such that $\pi(w z)=\pi(w)$ and $|z|=\pi(w)+\pi(u)-\rho(w)-1=$ $|w u|-|v|-1$. By Theorem 2, we have that $\pi(u)<\rho(w)$, and thus $x<\mathrm{p} u$. Now, $|v z|=|u x|-1$ and since $v z \leq_{\mathrm{p}} u x$, we have $v z=u x^{\bullet}=v y x^{\bullet}$, and thus $z=y x^{\bullet}$. Also, $z=x y^{\bullet}$, since $z \leq_{\mathrm{p}} u$ and $y<_{\mathrm{p}} u$, for, $y<_{\mathrm{p}} z<_{\mathrm{p}} u$ and $x$ is the root of $u$. By Lemma $1, y x^{\bullet}=x y^{\bullet}$ implies that there exists a primitive word $g$ such that $x=g^{i}$ and $y=g^{j}$ for some $i, j \geq 1$. Then $v=g^{i t} g_{1}$ for a prefix $g_{1}<_{\mathrm{p}} g$ and an integer $t \geq 0$, and so $u=v y=g^{i t} g_{1} g^{j}$. However, since $x$ is the root of $u, u=x^{r} x_{1}$ for some $r \geq 1$ and $x_{1}<_{\mathrm{p}} x$, from which it follows that $u=g^{i t+j} g_{1}$. In order for $g$ to be primitive, we must have $j=0$, for otherwise $g$ is a proper conjugate of itself. This contradicts the fact that $j \geq 1$. 
The bound given in Theorem 3 is optimal as shown in the following example.

Example 3 Consider the words

$$
w_{n}=(a b a)^{n} a b
$$

where $\pi\left(w_{n}\right)=3, \pi(u)=2$ for the root $u=a b a$ of $w_{n}$, and $\rho\left(w_{n}\right)=2$. Hence, $\kappa(w)=\pi\left(w_{n}\right)+\pi(u)-\rho\left(w_{n}\right)-2=1$. Indeed, the extension $w_{n} a b$ has a larger period than 3 , namely $\pi\left(w_{n} a b\right)=3 n+2$.

Also, for

$$
u_{n}=(a b)^{n} a a b
$$

of length $2 n+3$, we have $\pi\left(u_{n}\right)=2 n+1$, and the length $\rho\left(u_{n}\right)$ of the residue of $u_{n}$ is 2 . Hence, $\kappa\left(u_{n}\right)=2 n-1=\pi\left(u_{n}\right)+\pi\left((a b)^{n} a\right)-\rho\left(u_{n}\right)-2$.

\section{Critical points and extensions}

Every primitive word $w$ has an unbordered conjugate. For instance, consider the least conjugate of $w$ with respect to some lexicographic ordering, that is, a Lyndon conjugate of $w$; see e.g. Lothaire [8]. Denote by $\tau(w)$ the shortest prefix of $w$, $w=\tau(w) u$, such that the conjugate $u \tau(w)$ is unbordered. Hence $0 \leq \tau(w)<|w|$.

Lemma 2 Each primitive word $w$ has a factorization $w=u v$ such that the conjugate $v u$ is unbordered and either $|u|<\pi(w)$ or $|v|<\pi(w)$.

Proof Let $w=u^{k} z$, where $u$ is the root of $w, k \geq 1$, and $z<_{\mathrm{p}} u$. Suppose that $w$ has no conjugate as stated in the claim. Let $w^{\prime}=y u^{k-i} z u^{i-1} x$ be an unbordered conjugate of $w$, where $u=x y$. (Take, for instance, a Lyndon conjugate of $w$.) It follows that $i=k$ or $i=1$, for otherwise $y x$ is a border of $w^{\prime}$. If $i=1$, then $w^{\prime}=y u^{k-1} z x$ is a required conjugate: $w^{\prime}=\left(y u^{k-1} z\right)(x)$. Assume then that $i=k$, we have $w^{\prime}=y z u^{k-1} x$ and thus $z<_{\mathrm{p}} x$; otherwise again $y x$ is a border of $w^{\prime}$. However, now $w^{\prime}=(y z)\left(u^{k-1} x\right)$ is a required conjugate.

In the following we say that an integer $p$ with $1 \leq p<|w|$ is a point in the word $w$. A nonempty word $u$ is called a repetition word at $p$ if $w=x y$ with $|x|=p$ and there exist words $x^{\prime}$ and $y^{\prime}$ such that $u$ is a suffix of $x^{\prime} x$ and $u$ is a prefix of $y y^{\prime}$. Let

$$
\pi(w, p)=\min \{|u| \mid u \text { is a repetition word at } p\}
$$

denote the local period at point $p$ in $w$. In general, we have that $\pi(w, p) \leq \pi(w)$. A factorization $w=u v$, with $u, v \neq \varepsilon$ and $|u|=p$, is called critical, and $p$ is a critical point, if $\pi(w, p)=\pi(w)$.

The Critical Factorization Theorem (CFT) is a fundamental result on periodicity. It was first conjectured by Schützenberger [12] and then proved by Césari and Vincent [2]. Later it was developed into its present form by Duval [5]. We refer to [7] for a short proof of the theorem giving a technically improved version of the proof by Crochemore and Perrin [3].

Theorem 4 (CFT) Let $w$ be a word with at least two different letters. Then $w$ has a critical point $p$ such that $p<\pi(w)$. 
The following lemma rests on the CFT.

Lemma 3 Let $w$ be an unbordered word with $|w| \geq 2$, and let $w=u v$ be such that $p=|u|$ is any critical point of $w$. Then also the conjugate vu is unbordered.

Proof Without loss of generality we can assume that $|u| \leq|v|$. Now $\pi(w)=|w|$, since $w$ is unbordered. Assume, contrary to the claim, that the word $v u$ is bordered. We have two cases to consider. (1) Assume that $v=s v^{\prime}$ and $u=u^{\prime} s$ for a nonempty word $s$. Then $\pi(w,|u|) \leq|s|<|w|$ contradicting the assumption that $|u|$ is a critical point. (2) Assume that $v=s u t$. Then $\pi(w,|u|) \leq|s u|<|w|$, and again $|u|$ is not a critical point; a contradiction. These cases prove the claim.

The following theorem states the main result of this section.

Theorem 5 Let $w$ be a primitive word. Then $\tau(w)<\pi(w)$.

Proof Suppose first that $\pi(w)>|w| / 2$. Assume that $w=x y z$, where $|x y|=\pi(w)$, $z<_{\mathrm{p}} x y$, and $|x|$ is a critical point of $w$ such that $|x|<\pi(w)$ provided by Theorem 4 . Suppose that the conjugate $w^{\prime}=y z x$ is bordered, and let $u$ be its shortest border. Since $|x|$ is a critical point in $w$ and $u$ is a local repetition at $|x|$ in $w$, we have $|u| \geq$ $\pi(w)$, and hence $|u| \geq|y x|$. Since $u$ is unbordered, it does not overlap with itself, and therefore $|y z x| \geq 2|u|$, which implies that $|y z x| \geq 2|y x|$ and hence $|z| \geq|y x|$; a contradiction. Hence the conjugate $w^{\prime}=y z x$ is unbordered, and so $\tau(w)<\pi(w)$.

Assume then that $\pi(w)<|w| / 2$, and et $u$ be the root of $w$. Then $w=u^{k} z$ where $\pi(w)=|u|$ and $z<_{\mathrm{p}} u$ and $k \geq 2$.

Assume that $\tau(w) \geq \pi(w)$, and thus that $\tau(w)>\pi(w)$. By Lemma 2, there exists an unbordered conjugate $w^{\prime}=v u^{k-1} t$ of $w$, where $v \leq_{\mathrm{s}} w$ such that $|v|<$ $\pi(w)$. Consider a critical point $p$ of $w^{\prime}$, say $w^{\prime}=g h$, where $|g|=p$.

First, $v$ is a suffix of $u z$, and thus the critical point $p$ is not in $v$, i.e., $p>|v|$, since $\pi\left(w^{\prime}\right)=\left|w^{\prime}\right|$ and $v$ occurs in $u^{k-1} t$. Similarly, $p<|v u|$, since all suffixes of $w^{\prime}$ starting from a position $q \geq|v u|$ occur in $w^{\prime}$ starting from the point $q-|u|$ and thus there is a local repetition at point $q$ of length at most $|u|$. Now we have $|v|<|g|<|v u|$ and the conjugate $h g$ is unbordered by Lemma 3. Let $u=r s$ such that $g=v r$. Then $h g=s u^{k-1} z r$ and $1 \leq|r|<|u|$ as required.

The following example illustrates that it is not enough to just consider critical points for proving Theorem 5 .

Example 4 It is not true that a conjugate $v u$ with respect to a critical point $|u|$ of $w=u v$ is unbordered. Consider for instance the word $w=a b c b a b a b c b a b a b$, where $\pi(w)=6$, and $p=3$ is a critical point, but the corresponding conjugate $w^{\prime}=$ bababcbabababc has a border $b a b a b c$.

Note that we always have $\pi\left(w^{k} z\right) \leq|w|$ for prefixes $z \leq_{\mathrm{p}} w$ and nonnegative integers $k$. Theorem 5 gives a complementary result to Theorem 2 and 3 .

Corollary 2 Let $w$ be a word with a nonempty residue and a prefix $z \leq_{\mathrm{p}} w$.

$$
\text { If }|z| \geq \pi(w) \text { then } \pi(w z)=|w| .
$$


Proof Let $|z| \geq \pi(w)$. By Theorem 5, w has an unbordered conjugate $w^{\prime}=v u$ where $w=u v$ and $|u|<\pi(w)$. Then we have $\pi(w u)=|w|$ for the extension $w u$, since $\pi(w u)$ is at least the length of the longest unbordered factor of $w u$. The claim follows now from $w u \leq_{\mathrm{p}} w z$.

The following example elaborates on the differences between Theorem 2 and Corollary 2.

Example 5 Consider the word

$$
w=a a a b a a
$$

for which $|w|=6$ and $\pi(w)=4$ and $\operatorname{gcd}(\pi(w),|w|)=2$ so that we get $\pi(w)-$ $\operatorname{gcd}(\pi(w),|w|)=2$. We have $\pi(w z)>\pi(w)$ for each extension $w z$ with $z \leq_{\mathrm{p}} w$ and $|z| \geq 2$, by Theorem 2 . The shortest extension increasing the period is for $z=a a$, that is, $w \cdot a a=a a a b a a a a$ with $\pi(w a a)=5$.

However, we have $\pi(w z)<|w|$ and the corresponding conjugate $w^{\prime}=$ abaaaa of $w$ is bordered. In this example, we need an extension $z=a a a$ of length 3 in order to obtain $\pi(w z)=|w|$.

\section{References}

1. Berstel, J., Karhumäki, J.: Combinatorics on words - A tutorial. Bull. EATCS 79, 178-229 (2003)

2. Césari, Y., Vincent, M.: Une caractérisation des mots périodiques. C. R. Acad. Sci. Paris Sér. A 286, 1175-1177 (1978)

3. Crochemore, M., Perrin, D.: Two-way string-matching. J. ACM 38(3), 651-675 (1991)

4. Crochemore, M., Rytter, W.: Squares, cubes, and time-space efficient string searching. Algorithmica 13(5), 405-425 (1995)

5. Duval, J.P.: Périodes et répétitions des mots de monoïde libre. Theoret. Comput. Sci. 9(1), 17-26 (1979)

6. Fine, N.J., Wilf, H.S.: Uniqueness theorem for periodic functions. Proc. Amer. Math. Soc. 16, 109-114 (1965)

7. Harju, T., Nowotka, D.: Density of critical factorizations. Theor. Inform. Appl. 36(3), 315$327(2002)$

8. Lothaire, M.: Combinatorics on Words, Encyclopedia of Mathematics, vol. 17. AddisonWesley, Reading, MA (1983. Reprinted in the Cambridge Mathematical Library, Cambridge Univ. Press, 1997)

9. Lothaire, M.: Algebraic Combinatorics on Words, Encyclopedia of Mathematics and its Applications, vol. 90. Cambridge University Press, Cambridge, United Kingdom (2002)

10. Lothaire, M.: Algorithmic Combinatorics on Words. Cambridge University Press, Cambridge, United Kingdom (2005)

11. Morita, H., van Wijngaarden, A.J., Vinck, A.J.H.: On the construction of maximal prefixsynchronized codes. IEEE Trans. Inform. Theory 42, 2158-2166 (1996)

12. Schützenberger, M.P.: A property of finitely generated submonoids of free monoids. In: Algebraic theory of semigroups (Proc. Sixth Algebraic Conf., Szeged, 1976), Colloq. Math Soc. János Bolyai, vol. 20, pp. 545-576. North-Holland, Amsterdam (1979) 